\title{
MicroscopyEducation
}

\section{NanoGrande: Electron Microscopy Education and Outreach Through a Collaboration of Scientists and Artists}

\author{
P. Catravas, ${ }^{1 \star}$ K. Bubriski, ${ }^{2}$ M. D. Frey, ${ }^{3}$ M. E. Hagerman, ${ }^{4}$ \\ B. Cohen, ${ }^{5}$ J. J. McGee, ${ }^{6}$ and S. S. Bowser ${ }^{7}$ \\ ${ }^{1}$ Electrical and Computer Engineering Department, Union College, 807 Union Street, Schenectady, NY 12308 \\ ${ }^{2}$ Department of Visual Arts, Green Mountain College, One Brennan Circle, Poultney, VT 05764 \\ ${ }^{3}$ Center for Integrated Electronics, Rensselaer Polytechnic Institute, 110 Eighth Street, Troy, NY 12180 \\ ${ }^{4}$ Chemistry Department, Union College, 807 Union Street, Schenectady, NY 12308 \\ ${ }^{5}$ Biology Department, Union College, 807 Union Street, Schenectady, NY 12308 \\ ${ }^{6}$ Capital District Microscopy \& Microanalysis Society (www.cdmms.org) \\ ${ }^{7}$ Wadsworth Center, New York State Department of Health, Empire State Plaza, P.O. Box 509, Albany, NY 12201 \\ *catravap@union.edu
}

\section{Introduction}

NanoGrande is the culmination of an art-science effort that brought undergraduate students and faculty from science, engineering, and the visual arts together with professional microscopists of the Capital District Microscopy and Microanalysis Society for electron microscopy education and outreach. Students from two independent undergraduate courses, an advanced photography course and a microscopy laboratory course, collaborated on the project. The participants represented a wide range of majors, including chemistry, biology, electrical engineering, computer engineering, mechanical engineering, bioengineering, psychology, neuroscience, sociology/social sciences, history, and the visual arts. Emphasis was placed on both the scientific and the artistic aspects of the imaging process. The creation of electron microscopy images that were at the same time scientifically meaningful and visually compelling depended critically on communication of insights and ideas between paired students. The collaboration generated an artscience exhibition, NanoGrande, that has been presented to over four-thousand $\mathrm{K}$ through 12 students.

\section{Description of the Collaborating Courses}

Students enrolled in the electron microscopy course spent six weeks learning about the operating principles of the scanning electron microscope (SEM). They received tutorialstyle instruction in microscope operation, each working with another student, and completed hands-on exercises that covered the following topics: electron gun parameters and alignment, the effect of beam parameters and electron optics on image formation and optimization (including beam energy, spot size, working distance, interaction volume, and mitigation of artifacts), and sample preparation techniques. The laboratories were equipped with a Zeiss EVO-50 variable-pressure SEM and a Bruker energy-dispersive X-ray spectrometer (EDS) system. The majority of the exercises used the secondary electron detector, although there was one session devoted to $\mathrm{X}$-ray analysis and one session for comparing high-vacuum and variable-pressure modes of operation. Image artifacts were discussed and demonstrated throughout the exercises. The microscopy laboratory operated in parallel with a nanotechnology course covering semiconductor nanoparticles, carbon nanotubes, nanocomposites, bionanomaterials, and nanoelectromechanical materials.

In the meantime, across campus, photography students were learning about the use of analog black and white film and the creative craft of making fine art with gelatin silver photographic prints in the traditional wet darkroom. Students studying analog photography must learn to make choices and decisions at the time of the film's original exposure that will give visually compelling results. This is a skill that the great photographer of American landscapes, Edward Weston, called "pre-visualization." At the beginning of the semester, the analog photography students each received ten rolls of film. Their entire output of exposures for the semester was to be limited to only those three hundred and sixty frames of film. The photography students spent hours in the darkroom, often working with one negative, to make a print that most closely expressed the tonality they felt the photograph deserved. Photographers often "dodge" or "burn" areas of the image to heighten or lessen highlights and shadows in the final print. Ansel Adams expressed this process well by comparing photographic representation to music when he said that the negative is the score, but the print is the performance.

\section{Description of the Joint Project}

The microscopy students and photography students formed teams of two for the final four weeks of the term and imaged samples used in the nanotechnology course, including carbon nanotubes, electrospun fibers, and clay nanocomposites with embedded quantum dots, as well as samples from everyday life (bees, sandpaper, etc.). As a pedagogical exercise intended to emphasize the importance of applying their "pre-visualization" skills when acquiring SEM images, the students were not allowed to reframe the images using image processing software 


\section{Preparation Equipment and Microscopy Supplies}

The single source for All your microscopy supplies and specimen preparation equipment.

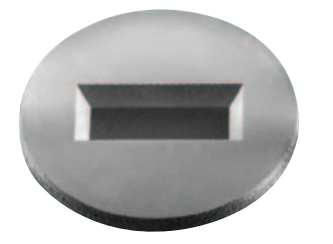

- Vacuum Coating Systems

- Calibration Standards

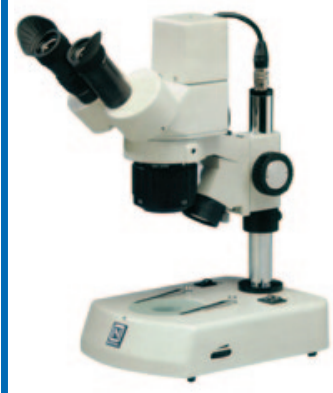

- PELCO ${ }^{\circledR}$ easiGlow ${ }^{\text {Tw }}$ Glow Discharge Unit

- SEM Sample Holders and Mounts

- Silicon NitrideTEM Membranes

- PELCO BioWave Pro ${ }^{\circledR}$ Tissue Processor

- TEM Support Films

- AFM Supplies

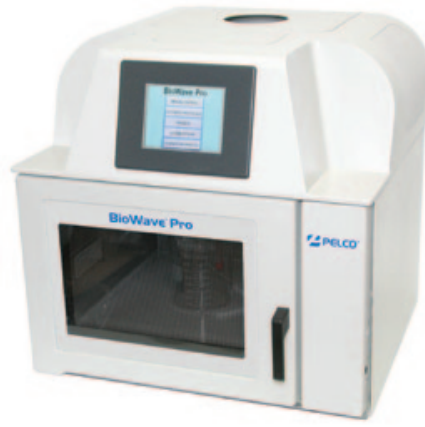

- Quality Laboratory Tweezers

- Vacuum Pick-up Systems

- Digital Stereo Microscopes

- Conductive Adhesives

- FIB Supplies
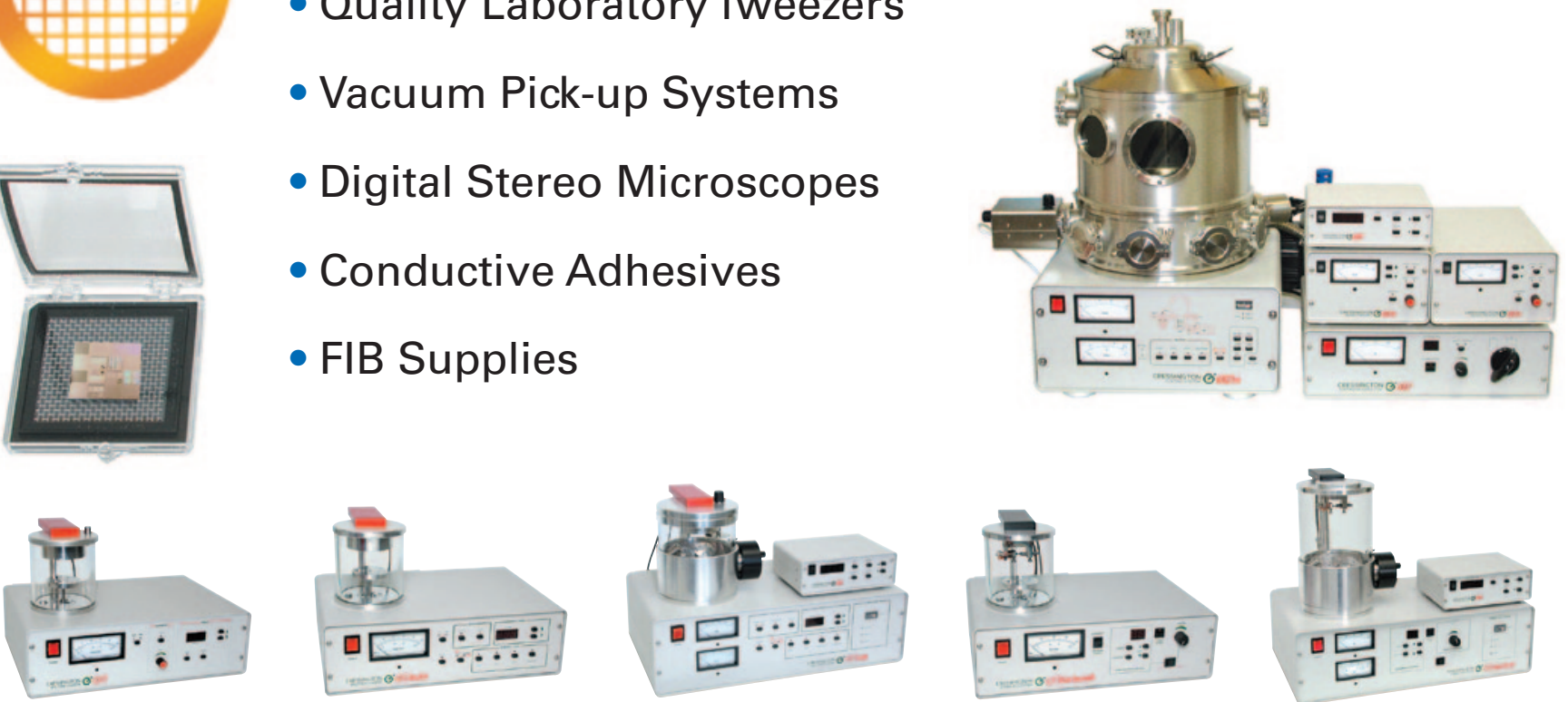

Complete line of compact Cressington EM Sample Coaters.

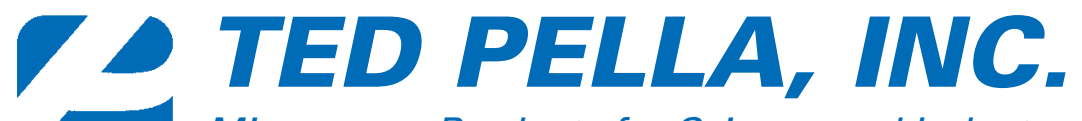

Microscopy Products for Science and Industry 
acquisition. Students from each team selected one SEM image to be printed in large format $(3 \mathrm{ft} \times 4 \mathrm{ft})$ for entry into the end-of-term competition.

\section{The Competition}

Eighty people attended the competition and public event that occurred in the final days of the course. The competition was juried by professionals from two groups: artists from the art faculty and microscopists from the Capital District Microscopy and Microanalysis Society (CDMMS). The evening event included a lecture on electron microscopy and art-science by CDMMS member S. Bowser and a talk by documentary photographer K. Bubriski, who taught the photography course.

A group of CDMMS members and two members of Union College's art department interviewed the twenty student teams. Each team entered one image in the contest. Two sets of winning images were selected independently by the two professional groups (microscopists and artists). Both groups provided feedback to the students: the microscopists discussed imaging technique and sample preparation, and the artists provided comments on composition and lighting issues.

The CDMMS judges ranked the images based on a set of specific criteria: 1 . Technical content: originality of the micrograph in relation to the work performed, 2. Photographic quality: aesthetic quality of the image and presentation, and 3. Specimen quality: appropriateness, uniqueness, and execution of the preparation technique. The microscopists assigned to each image a numerical score from 1 to 5 for each of the three criteria, and the results were tabulated to determine their six winning images. The two faculty members from Union College's art department chose a separate set of six winning images without a numerical scoring system. Interestingly, two of the winning images were the same across the two sets of judges.

Microscopists and artists provided constructive criticism on the images. For example, Figures 1 and 2 show two of the winning images. Microscopist David Frey provided the following commentary on the images:

The Metal Fragment: The composition of this image is such that it does a good job of drawing the viewer's eye

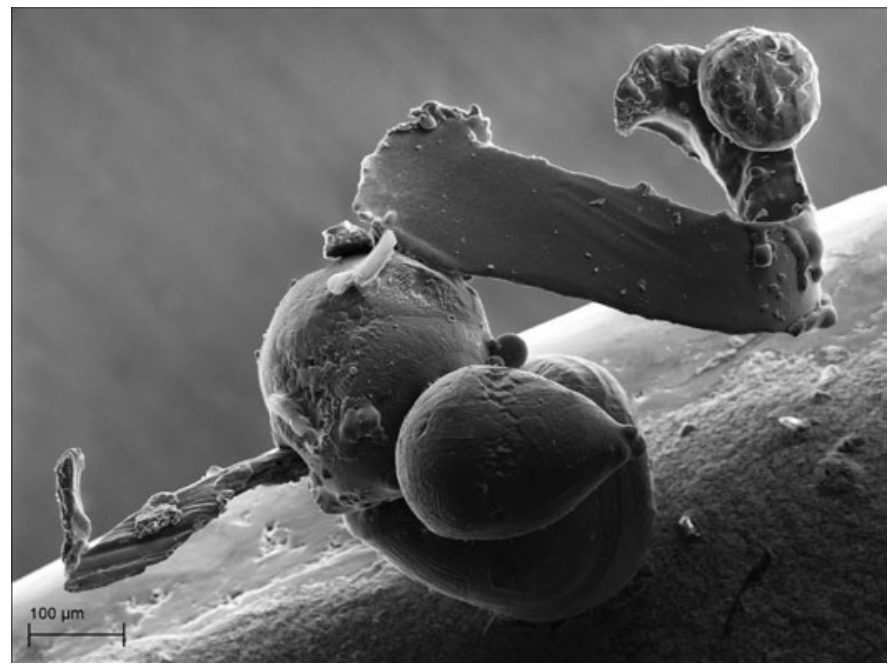

Figure 1: A small metal fragment from a building construction site. The image was created by a pair of undergraduate students from concurrent courses in nanotechnology and photography.

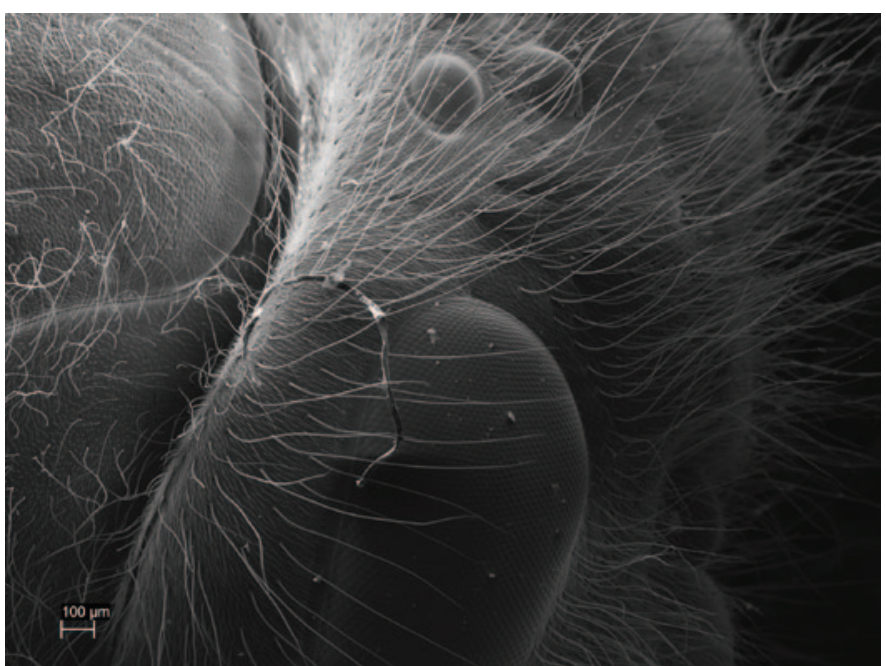

Figure 2: A close-up image of the eye of a bee. The image was created by a pair of undergraduate students from different fields.

from one edge to the other edge of the image. The background of the image contains no distracting elements and has a softness that keeps the viewer focused on the subject of the image. The micrograph is well focused and presents many different textures as the viewer examines the image with a closer eye. The grayscale is well balanced, but there are places where there is an apparent saturation of white or black that would lead to interpretation as a loss of image data or information. The microscope parameters seem to have been well selected and properly adjusted so that EHT (Extra High Tension or acceleration voltage), spot size, and depth of field helped to render an excellent image of subject matter.

The Bee's Eye: The overall quality of this image is good but a bit lower than that of the metal fragment. The framing of the image does not use the available area for imaging nearly as well as the metal fragment. The viewer's eye is primarily and only initially drawn along the $y$ axis of the image and then drawn to the left side of the image. This is the result of the sharpest focus in the image being along the left edge of the image. From a microscopist's point of view, after an initial examination of the focused area, the eye is drawn toward the right side of the image to the region that is beyond the depth of focus: the area of the image where image information is available but is overshadowed by the transition from sharp focus to out of focus. The grayscale contained within the image is somewhat compressed and is confined to the middle of the available range. This doesn't catch the eye of the viewer in such a way as to capture the attention of the examiner to further dissect the subject matter. Though the image is good, different choices regarding sample orientation, working distance, and magnification might have rendered an image with significantly more appeal. The small fibers across the eye of the bee also distract from the portrayal of the subject matter.

Documentary photographer Kevin Bubriski described the artist's point of view as follows: 
The Metal Fragment has been captured in a way that, to an imaginative eye, it appears as an organic living embryonic form with skeletal appendages coming from its right and left side. We lose a sense of scale as we try to place this metal fragment into the context of what we know through what we have seen with our naked eye. The image has been captured in such a way that there appears to be a surface that diagonally cuts across the frame. The metal fragment in its asymmetrical counterpoint is precariously balanced on this tilting horizon. The critical focus is sharp on the metal fragment, which lets our eyes feast on the rounded and jagged edges of the object, which is masterfully framed so that the appendages of the object fit snugly into the top-right and lower-left corners of the frame.

The Bee's Eye brings us in such close proximity to the surface and texture of the bee that it appears we are viewing a landscape as if from an aerial perspective or as if viewing a vertical surface of boulders and vegetation. The white fibers of the eye appear as seedlings or plants protruding from the landscape. Beneath the vibrant texture of the white fibers are the rounded forms of the eye surface. While the conscious science mind tells us we are looking at a bee's eye, the imagination wants to make sense of the image as perhaps strands of grass struggling on an ancient lava or rock surface, perhaps somewhere in Death Valley. The framing is so well done that the eye continues to explore all parts of the frame, starting with the white strands then exploring the multifaceted beautiful rounded forms just below the attention-grabbing white strands. The deep furrow or valley that runs vertically through the left half of the image attracts our eye with its bright tonalities and seductive curve. Then the eye travels to the right side of the frame to discern the subtle curves and shapes that loom in relative darkness and obscurity.

In addition to the prints, a slide show of over 100 images was created out of favorite images acquired by all the groups. Figure 3 shows a selection of these images. This slide show ran during the public and outreach events.

The art-science component transformed the course pedagogically. The plethora of unexpected requests from the photography students for adjustments to the images introduced a method for reinforcing the basic principles of SEM operation that would have been hard to achieve with a more traditional linear execution of a set of pre-defined laboratory exercises. The cumulative time needed over the term for the preparatory laboratories and acquisition of the final twenty competition images was about 200 hours. For six weeks, the microscopy students received one hour per week of handson instruction (in groups of two) that covered basic principles of SEM operation. For the final few weeks of the ten-week course, they were joined by the photography students, and each team was given a block of about three hours per week in which to acquire their image for competition entry. It was in the preparation of this final image that we observed the students acquire a fluency in applying the basic principles of SEM operation that was significantly improved over previous offerings of the course. Students were in the lab on some nights until 11 p.m. and for long hours on the weekend. Sometimes we could not get them to leave. They really looked forward to the public exhibition and to receiving feedback from the professional microscopists and artists. The sense of professionalism exhibited by the students, both in working with their peers and in presenting to the jury, was superb. Their enthusiasm was infectious. The documentary photographer was fascinated by the parallels between B/W
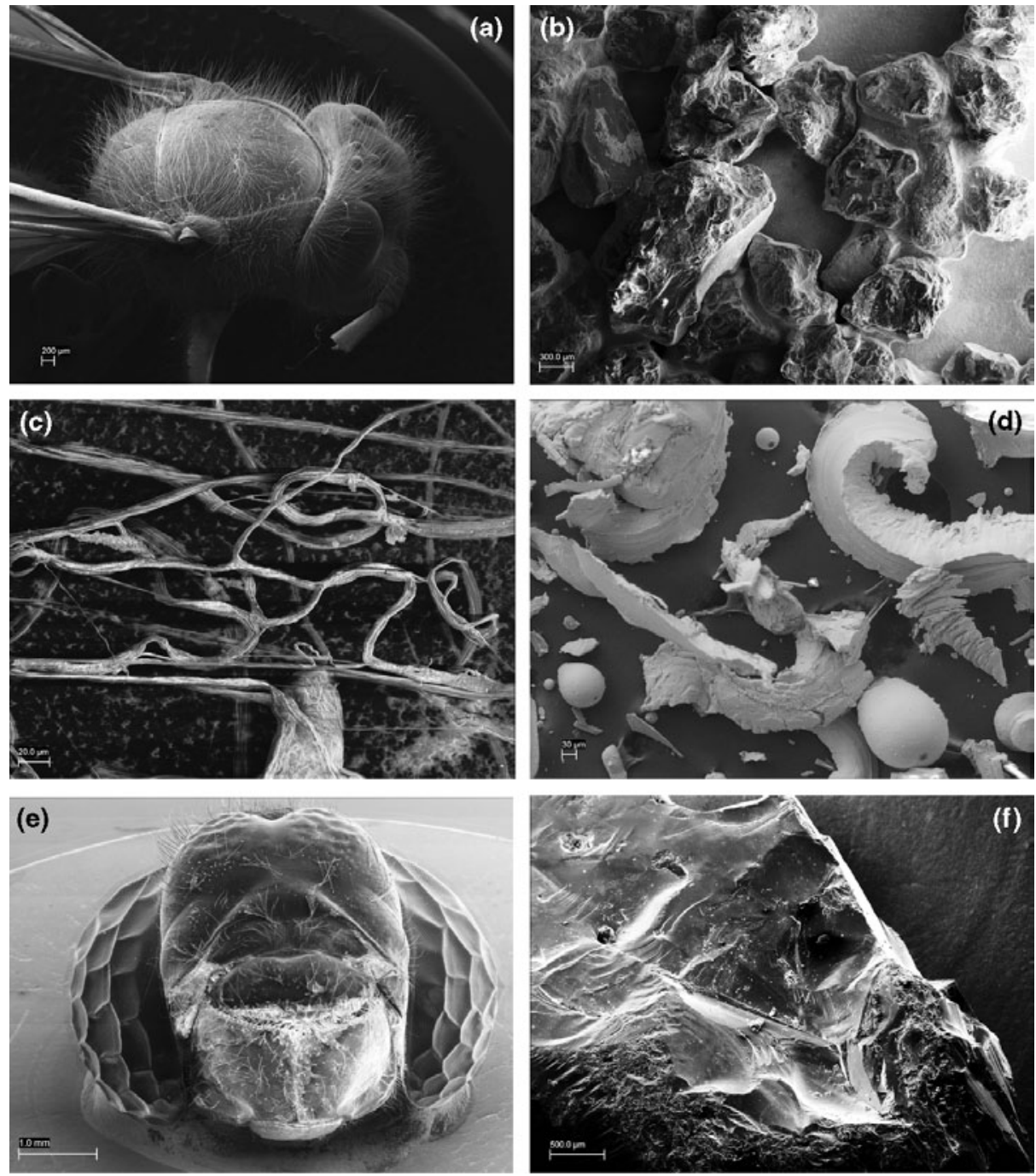

Figure 3: A montage of student work. (a) bee, field width $=8.32 \mathrm{~mm}$; (b) volcanic material, field width = $4.53 \mathrm{~mm}$; (c) electrospun fibers, field width = $327 \mu \mathrm{m}$; (d) construction debris, field width = $1.07 \mathrm{~mm}$; (e) dragonfly head, field width $=7.14 \mathrm{~mm}$; and $(\mathrm{f})$ a Herkimer diamond, field width $=4.67 \mathrm{~mm}$. 
photography and B/W electron microscopy. He embarked on an independent study the following term in which he learned to use the SEM in order to explore electron microscopy from the point of view of a photographer.

\section{The NanoGrande Exhibition and Outreach}

Following the competition, a traveling exhibition titled NanoGrande was generated from selected student work. (See Figure 4.) Twelve high-quality prints were created by Cone Editions Press in East Topsham, Vermont, using a special printing process that was especially well suited to the printing of black and white SEM images. The process, called Piezography ${ }^{\circledR} \mathrm{K} 7$, is a pure monochromatic inkjet process that had been developed by Cone Editions. All of the color inks in an Epson printer were replaced with seven progressive shades of pure carbon black ink. These Piezography inks are controlled with a special printer driver called QuadTone RIP. The inks are linearized using the Piezography inks profiler. In this way, the printer is effectively converted into a no-compromise monochromatic process for fine black and white photography. In fact, the 16-bit printing process resolved certain details in the SEM images that had not been detected prior to printing. The people at Cone Editions worked to create prints that were stylistically reminiscent of those of classic black-and-white photographers. It was in the printing process that reflections of Ansel Adams's quote emerged, where the electron microscope image had become the score and the 16-bit black and white prints the performance.

The images taken by the college students were also incorporated into two community outreach programs. First, students from the STEP program (Science and Technology Entry Program), organized by the Kenney Center at Union College, were invited to attend the competition and public event. The images subsequently provided the scientific connection for the summer STEP program icebreaker event, attended by about seventy middle school and

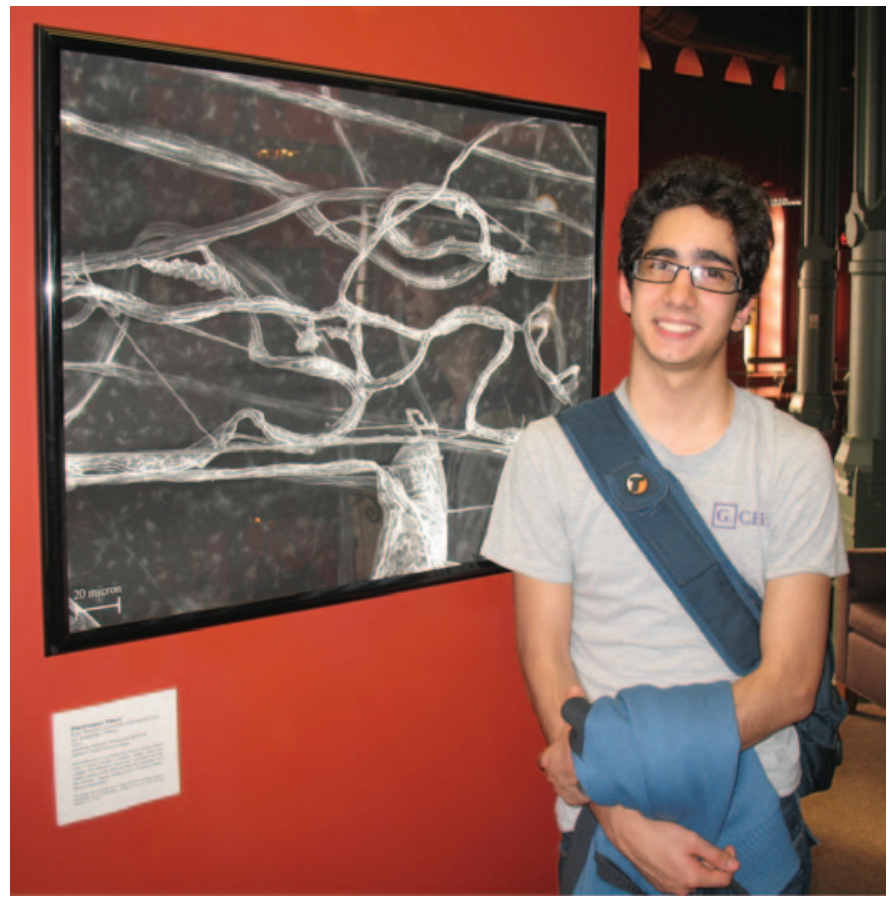

Figure 4: One of the microscopy students, Amin Meyghani, stands by his print at the NanoGrande exhibition in the Wikoff Gallery, Union College (April, 2010). high school students. The younger students were given a set of magazine clippings and one SEM image and asked to generate a story. In a second outreach effort, the NanoGrande traveling exhibition was displayed in the Wikoff Gallery at Union College for several weeks. Subsequently it was exhibited at the Gateway Science Museum in Chico, CA, where it was presented to over $4,000 \mathrm{~K}$ through 12 students as part of organized field trips. To inquire about use of the traveling exhibit, please contact Palma Catravas at catravap@union.edu.

\section{Conclusion}

This project brought science and art together under controlled conditions. It provided an opportunity for microscopy and photography students to work in teams and combine their knowledge of physics and aesthetics. Importantly, it culminated in a meaningful dialog about scientific images and how to present them. Finally, the project produced interesting large-format images that were used to excite K-12 students about art and science.

\section{Acknowledgments}

We thank the students in the Frontiers of Nanotechnology (ESC224/CHM224) and Advanced Photography (AVA220) courses at Union College who acquired the images shown in the figures. Support from Jon and Cathy Cone at Cone Editions Press in Topsam, VT; Ed Whalen from A. J. Martini Inc.; Rachel Teasdale and Tiffany Fisher at the Gateway Science Museum at the California State University, Chico; and the National Science Foundation NSF NUE \#0939322 and ANT \#0739583 are gratefully acknowledged.

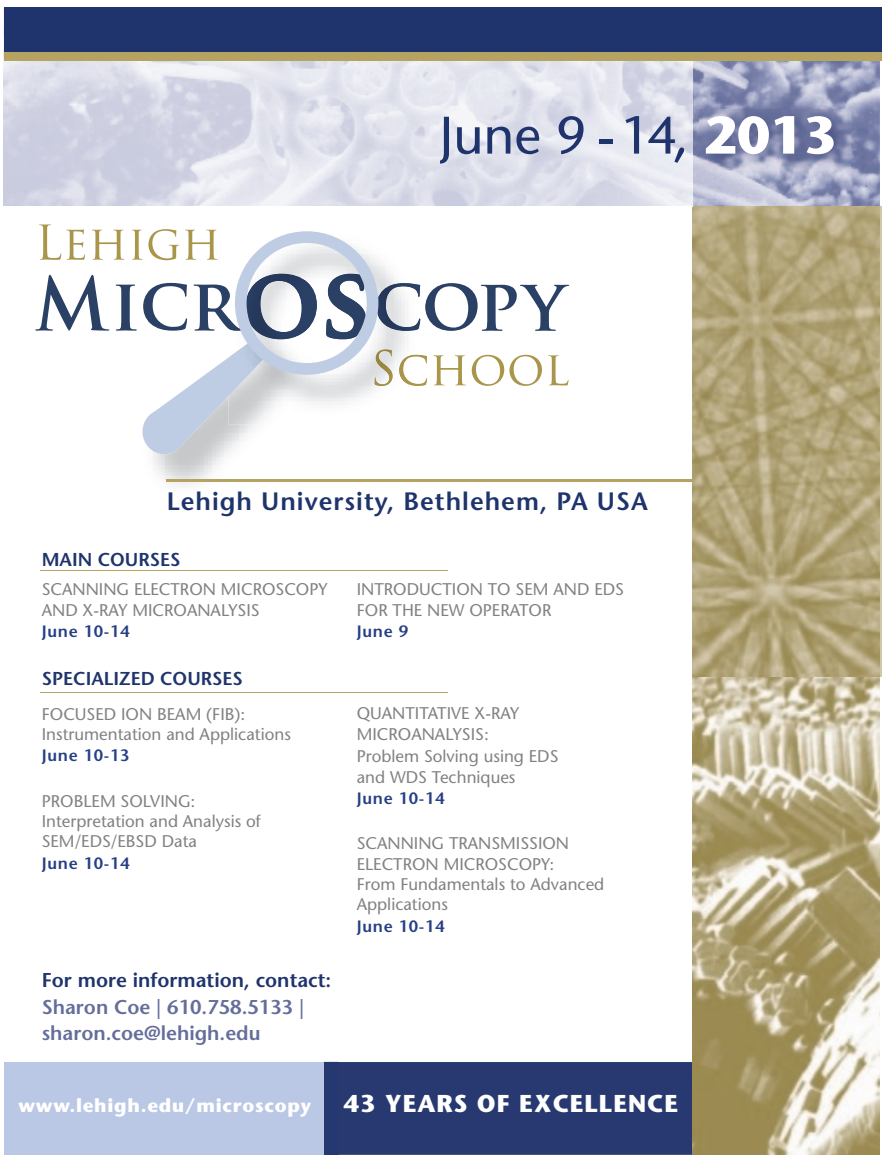




\title{
Time for a change?
}

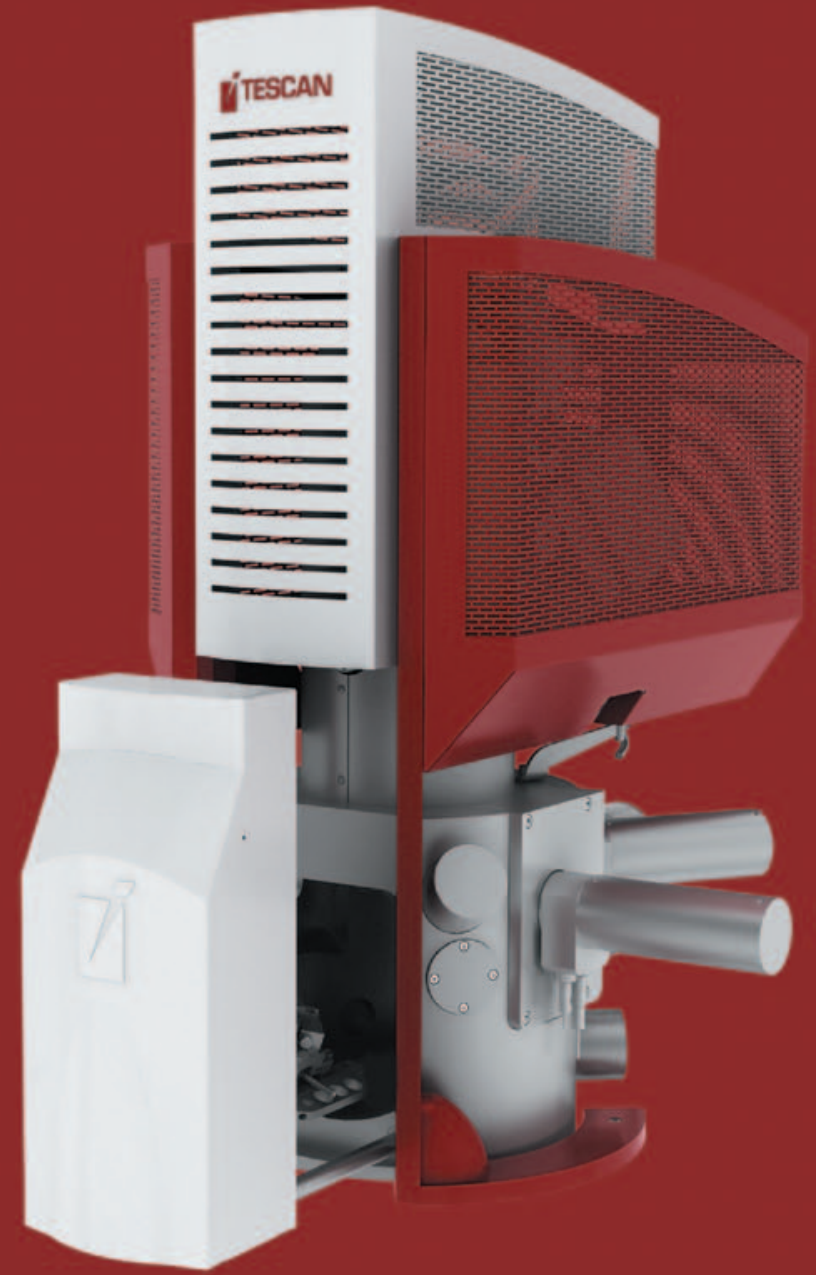

Reliability, performance, superior analytical flexibility and ease of use sums up the new generation MIRA3 FE SEM from TESCAN.

\author{
Come visit us and find out why \\ more and more researchers are choosing TESCAN.
}

*Thermionic Scanning Electron Microscopes

*Field Emission Scanning Electron Microscopes

*FIB-SEM Workstations

*FIB-SEM Time of Flight Integration

*Plasma FIB-SEM Workstations

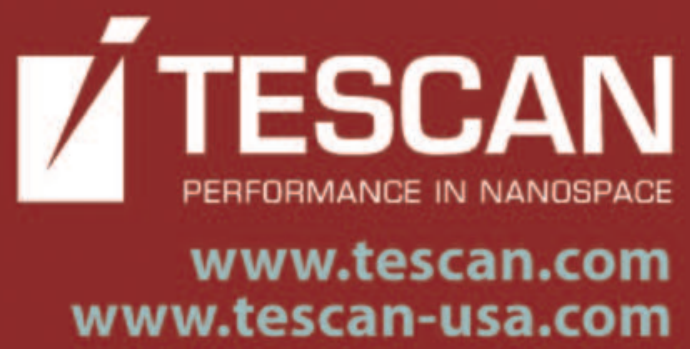

